economic variance. Therefore other characteristics like GP use (particularly for mental health reasons) and psychosocial wellbeing become more important in explaining PASB. Future longitudinal research is planned to explore causation-for example, do GP users worry about teenagers because illness encourages feelings of vulnerability-or does worrying about neighbourhood problems exacerbate ill health? Current findings are consistent with the view that addressing PASB be included in strategies to address health inequalities.

\section{Alcohol

075 GAMMA-GLUTAMYLTRANSFERASE AS A PREDICTOR FOR
ALCOHOL- AND NON-ALCOHOL-RELATED CANCER
INCIDENCE

doi:10.1136/jech.2010.120956.75

${ }^{1} \mathrm{~S}$ Strohmaier, ${ }^{1} \mathrm{~W}$ Borena, ${ }^{1} \mathrm{~A}$ Strasak, ${ }^{1} \mathrm{G}$ Goebel, ${ }^{1} \mathrm{M}$ Edlinger, ${ }^{2} \mathrm{G}$ Diem, ${ }^{2} \mathrm{H}$ Concin, ${ }^{3} \mathrm{C}$ Kelleher, ${ }^{1} \mathrm{H}$ Ulmer. ${ }^{1}$ Department for Medical Statistics, Informatics and Health Economics, Innsbruck Medical University, Innsbruck, Austria; ${ }^{2}$ Agency for Preventiveand Social Medicine, Bregenz, Austria; ${ }^{3}$ School of Public Health and Population Science, University College Dublin, Dublin, Republic of Ireland

Objective Recent evidence suggests that elevated levels of gammaglutamyltransferase (GGT) are associated with both incidence and mortality of cardiovascular disease and cancer. Although GGT is regarded as a marker of liver function which may in turn reflect alcohol consumption, to date, no study has investigated the relationship of GGT with cancer sites known to be alcohol-related or non-related.

Design Prospective cohort follow up and linkage study.

Participants and setting First visit measurements in 94628 adult women and 80224 men screened for metabolic risk factors as part of a standardised primary care assessment in Vorarlberg province of Austria. During a median follow-up of 13 years, a total of 5136 incident cancers were diagnosed in men and 4665 in women.

Methods Sex-specific Cox proportional hazards models, adjusted for age, body mass index and smoking were performed to estimate HRs and $95 \%$ CI per quintiles of GGT.

Results In males, the highest GGT-quintile revealed a high risk of alcohol-related cancer incidence (HR 2.20, 95\% CI 1.74 to to 2.78). The association was strongest for cancers of the liver and intrahepatic bile ducts (HR 16.50, 4.00-68.19), followed by cancers of the lip, oral cavity, pharynx and larynx (HR 3.80, 2.33-6.20), esophageal cancer (HR 2.39, 1.01-5.72) and colorectal cancer (HR $1.36,1.01-1.83)$. In females, there was a modest but significant association between GGT and alcohol-related cancers (HR 1.16, 1.02-1.32). GGT showed a significant association in breast cancer only (HR 1.19, 1.02-1.39). HRs were clearly elevated for cancers of the liver and intrahepatic bile ducts and for cancers of the lip, oral cavity, pharynx, larynx and oesophagus, however, without reaching significance due to limited number of cases. No association was seen for colorectal cancer. Additionally, elevated GGT was found to be significantly related to cancers with weak or no evidence of alcohol consumption as a risk factor. In males, there were associations with pancreatic cancer (HR 2.13, 1.01-4.56), lung cancer (HR 2.04, 1.55-2.70), bladder cancer (HR 1.76, $1.11-2.77$ ) and kidney cancer (HR 1.61, 0.92-2.82, $\mathrm{p}$ for trend $=0.009$ ). In females, the association was most pronounced in cervical cancer (HR 3.77, 1.94-7.32), followed by lung cancer (HR $1.63,1.02-2.60$ ) and endometrial cancer (HR 1.42, 0.98-2.05, p for trend $=0.013$ ).

Conclusion Although elevated GGT levels were strongly associated with incidence of alcohol-related cancers, most markedly in men, there were still effects of GGT in non-alcohol related cancer sites.
This suggests that alcohol consumption explains the relationship between GGT and cancer outcomes only in part.

\section{BINGE DRINKING IN MIDLIFE AND THE RISK OF DEVELOPING DEPRESSION DURING YEARS OF FOLLOW-UP}

doi:10.1136/jech.2010.120956.76

S Bell, A Britton, M Shipley. Department of Epidemiology and Public Health, University College London, London, UK

Objective To examine the relationship between binge drinking at baseline and the onset of new depression during 24 years of followup after adjustment for age, socio-economic status, education and marital status.

Design and setting Data from phases 1 (1985-1988) to 9 (2007-2009) of the Whitehall II prospective cohort were used.

Participants 5985 (male $=4161$, female $=1824$ ) British civil servants aged 35-55 years who were free from depression at baseline.

Comparison groups Cohort members were grouped, at phase 1, according to their self-reported usual and maximum number of alcoholic drinks consumed in a single sitting-abstainers, nonbingers (reference category) and bingers. Alcohol consumption was split into two categories and number of drinks consumed was converted to units for analysis: wine and spirits ( 1 unit per drink), and beer ( 2 units per drink). For usual drinking sessions those who reported consuming $5+$ units of wine/spirits and $10+$ units of beer were categorised as bingers, those consuming 1-4 units of wine/ spirits or 1-9 units of beer were classified as non-bingers. For maximum drinking sessions, participants were defined as bingers following the Department of Health guidelines as those consuming $8+$ or $6+$ units of alcohol for males and females respectively for both categories of consumption. Those who reported consuming no drinks were classified as abstainers in all analyses.

Main outcome measures The 30-item General Health Questionnaire (GHQ-30) was administered at all phases of data collection. The depression subscale of the GHO-30 was used to identify new cases of depression (scores of 4 or more) across all phases.

Results Adjusted HRs and 95\% CIs were estimated using Cox proportional hazard models fitted in the total cohort and stratified by gender. Usual drinking session spirit/wine bingers had an elevated risk of depression (HR 1.28, CI 1.02 to to 1.60) compared to non-bingers in the total sample. Maximum drinking session spirit/ wine bingers had a greater risk of depression in the total (HR 1.23, CI 1.04 to to 1.44 ) and male (HR 1.27, CI 1.03 to to 1.56 ) samples. There were no statistically significant effects when using beer measures as exposures or for abstainers in any alcohol measures after adjustment for confounders.

Conclusion Binge drinking on wine and spirits, but not beer, in midlife increases the risk of having a depressive episode over the course of the following 22-24 year period. Future work will examine other covariates and explore bidirectional issues in this relationship.

\section{ALCOHOL USE AMONG RUSSIAN MEN: THE ASSOCIATION BETWEEN AUDIT SCORE AND SELF- AND PROXY-REPORTED DRINKING BEHAVIOURS}

doi:10.1136/jech.2010.120956.77

S Cook, B De Stavola, D Leon. London School of Hygiene and Tropical Medicine, London, UK

Objectives Alcohol use in Russia has a number of relatively distinct features which have been shown to be strongly associated with mortality. The aim of this paper is to investigate how specific 
aspects of Russian drinking behaviour and its consequences are related to scores obtained from the internationally recognised AUDIT instrument for detecting alcohol problems.

Design Population-based age-stratified cross sectional survey.

Setting Izhevsk, Russia.

Participants 925 men aged 25-59 years.

Main outcome measure Men and proxy respondents completed an interview including questions on alcohol consumption and socioeconomic and demographic variables. These included questions on six drinking behaviours and their consequences. These were: going on zapoi (a period of continuous drunkenness of two or more days); drinking non- beverage alcohol; frequency of excessive drunkenness; frequency of hangover; drinking before noon and frequency of sleeping in clothes at night because of drunkenness. The men also completed a Self Completed Questionnaire containing questions constituting the alcohol use disorders identification test (AUDIT)

Results There was a strong association with AUDIT score and hazardous drinking (one or more episodes of zapoi in the past year or drinking non beverage alcohol or twice a week or more occurrence of excessive drunkenness, hangover or going to sleep at night clothed because of drunkenness) using both proxy and self report of drinking behaviours (self-report OR 5.25 95\% CI 2.86 to to 9.65). The association was stronger when proxy report was used (proxy-report OR $6.6695 \%$ CI 4.00 to to 11.07). The same pattern was seen for all the drinking behaviours individually. Confirmatory factor analysis supported a two factor structure for the AUDIT measuring alcohol consumption and alcohol related problems. Hazardous drinking behaviour specific to Russia was more strongly associated with AUDIT questions relating to alcohol related problems than to questions measuring consumption.

Conclusions The drinking behaviours used in this paper are unconventional measures of hazardous drinking. The strong association with AUDIT shows that both self and in particular proxy report of distinctive directly observable drinking behaviours provide useful data on alcohol consumption and abuse in Russian men.

\section{Cancer incidence}

\section{INCIDENCE RATES AND SURVIVAL TRENDS OF CANCER IN 0-29-YEAR-OLDS BY ETHNIC GROUP IN YORKSHIRE, UK}

\section{doi:10.1136/jech.2010.120956.78}

${ }^{1} \mathrm{M}$ van Laar, ${ }^{1} \mathrm{P}$ A McKinney, ${ }^{1} \mathrm{R}$ C Parslow, ${ }^{2} \mathrm{~A}$ Glaser, ${ }^{2} \mathrm{~S}$ E Kinsey, ${ }^{2} \mathrm{~J}$ Lewis, ${ }^{2} \mathrm{~S} V$ Picton, ${ }^{2} \mathrm{M}$ Richards, ${ }^{2} \mathrm{G}$ Shenton, ${ }^{3} \mathrm{D}$ Stark, ${ }^{4} \mathrm{P}$ Norman, ${ }^{1} \mathrm{R}$ G Feltbower. ${ }^{1}$ Paediatric Epidemiology Group, Division of Epidemiology, University of Leeds, UK; ${ }^{2}$ Paediatric Oncology and Haematology, St James's University Hospital, Leeds, UK; ${ }^{3}$ Institute of Oncology, Bexley Wing, St James's University Hospital, Leeds, UK; ${ }^{4}$ School of Geography, University of Leeds, Leeds, UK

Objective Few studies have examined differences in the epidemiology of cancer between ethnic groups for children and young adults in the UK. We investigated incidence rates, associated trends and survival by ethnicity (south Asian vs all other ethnic groups) across childhood (0-14) and young adult (15-29) ages using a unique specialist cancer register.

Methods The data used for this study were extracted from the Yorkshire Specialist Register of Cancer in Children and Young People. Patients diagnosed from 1990 to 2005 in the former Yorkshire Regional Health Authority were included in the analysis. Ethnicity was assigned using name analysis programs and Hospital Episode Statistics data. Incidence rates (per 1000000 person-years) by ethnic group were derived using mid-year population estimates. Poisson regression was used to examine trends in incidence by ethnicity and diagnostic sub-group, adjusting for sex and age. An interaction term between year and ethnicity was added to the model and likelihood-ratio test used to determine whether incidence trends differed for south and non south Asians. Survival rates were assessed using Kaplan-Meier estimates and log-rank tests. Cox regression was used to assess the effect of ethnicity on survival, adjusting for age, sex, year and deprivation.

Results Overall cancer incidence was similar for south Asians (12.1; 95\% CI 10.7 to to $13.5, \mathrm{n}=275$ ) and non-south Asians (12.6; 95\% CI 12.2 to to $13.1, \mathrm{n}=3259)$. For non-south Asians, incidence rates increased on average by $1.5 \%$ per year ( $95 \%$ CI 0.8 to to 2.3 ); the rate of increase for south Asians was significantly higher (7.0\%; $95 \%$ CI 4.2 to to 9.9). Survival rates were significantly poorer for $15-29$ vs $0-14$ year olds (HR 1.25; 95\% CI 1.09 to to 1.43). A significant increased risk of death was seen for south Asians compared to nonsouth Asians with leukaemia (HR 1.76; 95\% CI 1.10 to to 2.81) and lymphoma (HR 3.11; 95\% CI 1.61 to to 5.99). South Asians with other solid tumours had a significantly reduced risk of death (HR 0.43 95\% CI 0.23 to to 0.81) compared to non-south Asians.

Conclusion If present trends continue, the higher rate of increase seen among the Asian population in Yorkshire will result in 3-times higher incidence than non-south Asians by 2020. Lower survival rates seen for south Asians with leukaemia and lymphoma and for 15-29 year olds warrant further detailed investigation.

\section{INCREASING INCIDENCE OF THYROID CANCER IN GREAT BRITAIN, 1976-2005: AGE-PERIOD-COHORT ANALYSIS}

doi:10.1136/jech.2010.120956.79

${ }^{1} \mathrm{R}$ J 0 McNally, ${ }^{1} \mathrm{~K}$ Blakey, ${ }^{1} \mathrm{P}$ W James, ${ }^{1} \mathrm{~B}$ Gomez Pozo, ${ }^{1} \mathrm{~N} 0$ Basta, ${ }^{2} \mathrm{~J}$ Hale. ${ }^{1}$ Institute of Health and Society, Newcastle University, Newcastle-upon- Tyne, UK; ${ }^{2}$ Northern Institute of Cancer Research, Newcastle University, Newcastle-upon- Tyne, UK

Objective To examine temporal trends in the incidence of primary thyroid cancers diagnosed in 0-49 year olds in parts of Great Britain (GB) during the period 1976-2005. We specifically aimed to analyse age, period and cohort effects

Design Population-based descriptive analysis of cancer registry data. Setting Parts of Great Britain.

Participants Case data on thyroid cancer were obtained from four regional cancer registries in GB (i. Northern and Yorkshire, ii. North West, iii. Wales and iv. Scotland).

Main outcome measures Age-standardised incidence rates (ASRs) and $95 \%$ CIs were calculated. Negative binomial regression was used to examine the effects of age, sex, drift (linear trend), non-linear period and non-linear cohort.

Results The study analysed 4327 cases of thyroid cancer aged 0-49 years at diagnosis. For males, the overall ASR was 3.9 per million persons per year (95\% CI 3.6 to to 4.1 ). For females, the overall ASR was 12.5 per million persons per year (95\% CI 12.0 to to 12.9). The best fitting negative binomial regression model included age $(p<0.001)$, sex $(p<0.001)$ and drift $(p<0.001)$. Non-linear period $(p=0.42)$ and non-linear cohort $(p=0.71)$ were not statistically significant. For males aged $0-14$ years, the ASR increased from 0.2 per million persons per year in 1976-1986 to 0.6 per million persons per year in 1997-2005. For males aged 15-29 years and 30-49 years the ASRs increased from 1.9 to 3.2 and from 7.3 to 12.6 per million persons per year, respectively. For females aged $0-14$ years, the ASR increased from 0.3 to 0.5 per million persons per year. For females aged 15-29 years and 30-49 years the ASRs increased from 7.0 to 12.3 and from 21.2 to 40.0 per million persons per year, respectively. Conclusions There has been a linear increase in the incidence of thyroid cancer, which has led to a doubling of the number of cases diagnosed over a 20 year time span. The reasons for this increase are not well understood, but it is consistent with findings from other countries 